\title{
Complementos alimentares acessíveis para idosos em risco nutricional
}

\author{
Accessible dietary supplements for elderly in nutritional risk \\ Complementos alimenticios asequibles para personas mayores con riesgo nutricional
}

Recebido: 09/06/2021 | Revisado: 19/06/2021 | Aceito: 22/06/2021 | Publicado: 07/07/2021

\author{
Andressa Bressan Pedroso \\ ORCID: https://orcid.org/0000-0002-7648-6268 \\ Universidade de São Paulo, Brasil \\ E-mail: nutridessapedroso@gmail.com \\ Giovana Flores Safraid \\ ORCID: https://orcid.org/0000-0002-6618-2340 \\ Universidade Federal de Santa Maria, Brasil \\ E-mail: g.safraid@gmail.com \\ Juliana Ebling Brondani \\ ORCID: https://orcid.org/0000-0001-5478-6633 \\ Hospital Universitário de Santa Maria, Brasil \\ E-mail: juebrondani@gmail.com \\ Giovana Cristina Ceni \\ ORCID: https://orcid.org/0000-0003-2787-7593 \\ Universidade Federal de Santa Maria, Brasil \\ E-mail: giovana.ceni@ufsm.br
}

\begin{abstract}
Resumo
O estado nutricional é um fator de relevância clínica na saúde do idoso. O objetivo do estudo foi desenvolver complementos alimentares acessíveis para idosos, bem como instrumento para aconselhamento nutricional. Trata-se de um estudo experimental, em que foram desenvolvidos complementos alimentares e preparações dietéticas fortificadas com os mesmos. As preparações foram elaboradas considerando as recomendações nutricionais atuais para suplementação em idosos, e foram testadas em uma cozinha hospitalar. A composição nutricional dos complementos foi calculada a partir das fichas técnicas, com auxílio de um software nutricional. O custo médio foi calculado com base nos valores de mercado de agosto a outubro de 2020. Foram desenvolvidos cinco complementos (preparações-base) e seis receitas, nas quais estes pudessem ser incorporados. Os complementos alcançaram recomendações calóricas e proteicas de suplementação para idosos, e junto às receitas fortificadas, adequaram-se a diabetes, disfagia e intolerância à lactose. Foi elaborada uma cartilha para aconselhamento nutricional no formato de livreto. Opções de complementos alimentares podem ser alternativas acessíveis para idosos em risco nutricional.
\end{abstract}

Palavras-chave: Envelhecimento; Desnutrição; Suplementos nutricionais.

\begin{abstract}
Nutritional status is a factor of clinical relevance in the health of the elderly. The aim of the study was to develop accessible food supplements for the elderly, as well as an instrument for nutritional counseling. This is an experimental study, in which food supplements and dietary preparations fortified with them were developed. The preparations were made considering the current nutritional recommendations for supplementation in the elderly, and were tested in a hospital kitchen. The nutritional composition of the supplements was calculated from the technical data sheets, with the aid of a nutritional software. The average cost was calculated based on the market values from August to October 2020. Five supplements (basic preparations) were developed, as well as six recipes in which they could be incorporated. The supplements achieved caloric and protein recommendations for supplementation for the elderly, and along with the fortified recipes, they were suitable for diabetes, dysphagia and lactose intolerance. A booklet for nutritional counseling was prepared. Homemade options for food supplements can be affordable alternatives for elderly people at nutritional risk.
\end{abstract}

Keywords: Aging; Malnutrition; Dietary supplements.

\section{Resumen}

El estado nutricional es un factor de relevancia clínica en la salud de las personas mayores. El objetivo del estudio fue desarrollar complementos alimenticios accesibles para personas mayores, así como un instrumento de asesoramiento nutricional. Se trata de un estudio experimental, en el que se desarrollaron complementos alimenticios y preparaciones dietéticas enriquecidas con ellos. Los preparados se elaboraron teniendo en cuenta las recomendaciones nutricionales actuales para la suplementación en ancianos y se probaron en la cocina de un hospital. La composición nutricional de los suplementos se calculó a partir de las fichas técnicas, con la ayuda de un software nutricional. El costo promedio se calculó en base a los valores de mercado de agosto a octubre de 2020. Se desarrollaron cinco suplementos 
(preparaciones básicas) y seis recetas, en las que estos podrían incorporarse. Los suplementos alcanzaron las recomendaciones calóricas y proteicas para la suplementación de los ancianos y, junto con las recetas enriquecidas, fueron adecuados para la diabetes, la disfagia y la intolerancia a la lactosa. Se preparó un folleto de asesoramiento nutricional en forma de folleto. Las opciones de complementos alimenticios pueden ser alternativas asequibles para las personas mayores con riesgo nutricional.

Palabras clave: Envejecimiento; Desnutrición; Suplementos Dietéticos.

\section{Introdução}

No envelhecimento ocorre acúmulo de danos moleculares e celulares, resultando em alteração na funcionalidade do corpo (WHO, 2017). O estado nutricional do idoso é um fator de relevância clínica visto que essa população pode apresentar risco nutricional (WHO, 2019; Horie et al., 2019). Ainda, a insegurança alimentar provoca desenvolvimento de incapacidades ao longo do envelhecimento (WHO, 2017). Neste contexto, a situação socioeconômica pode influenciar o estado nutricional de idosos (Silva et al., 2018).

Para indivíduos em risco de desnutrição, a Organização Mundial de Saúde orienta oferta de suplementação alimentar e aconselhamento nutricional (WHO, 2019). Outras organizações também ressaltam a importância da suplementação alimentar e da oferta de alimentos fortificados (Horie et al., 2019; Volkert et al., 2019).

Os suplementos alimentares são alimentos com maior concentração de nutrientes, utilizados para suplementar a dieta normal daqueles cuja ingestão não alcança as necessidades nutricionais (Cederholm et al., 2017; WHO 2019). Eles podem contribuir para a redução de complicações e mortalidade em idosos hospitalizados, reduzir o número de readmissões, promovendo aumento da ingestão calórica e proteica, do peso corporal e da força de preensão palmar (Elia et al., 2016; Volkert et al., 2019; Horie et al., 2019).

Em adição, o desenvolvimento das habilidades culinárias é considerado como um elemento promotor da alimentação saudável (Brasil, 2014). No estudo com uma população de idosos, de Curitiba - PR, Martins et al. (2021) observaram que os participantes demonstraram conhecer termos e técnicas culinárias, indicando habilidades culinárias e práticas alimentares positivas.

Tendo em vista essa problemática e considerando que o custo de suplementos alimentares industrializados pode ser fator limitante para a adesão às orientações nutricionais, o uso de opções de menor valor poderia garantir o alcance das necessidades nutricionais. Assim, o presente trabalho teve como objetivo desenvolver opções de complementação alimentar e de uma cartilha ilustrativa, para uso em aconselhamento de pacientes com desnutrição ou risco nutricional.

\section{Metodologia}

Pesquisa experimental com desenvolvimento de complementos alimentares. Calculou-se combinações-base de ingredientes capazes de complementar a oferta de calorias e de proteínas por dia, considerando as recomendações para suplementação de idosos (WHO, 2019; Horie et al., 2019; Volkert et al., 2019).

Foram formuladas preparações com as combinações base, considerando: custo; tempo de preparo; acesso aos ingredientes e composição nutricional. Os ingredientes foram selecionados levando em conta a composição nutricional, a sazonalidade, os custos e a disponibilidade. O custo dos complementos foi calculado utilizando valores de mercado entre agosto e outubro de 2020. As preparações foram desenvolvidas em um laboratório de técnica dietética. Os dados foram utilizados para a elaborada uma cartilha de uso na orientação nutricional dos pacientes e seus familiares/cuidadores.

Utilizou-se os programas Dietwin - Tabela Nutricional ${ }^{\circledR}$ (DietWin: Porto Alegre, Brasil) para análise nutricional. Para a cartilha foi utilizado o software Adobe Illustrator $\mathrm{CC}^{\circledR}$ (Adobe Inc.: San Jose, Estados Unidos). 


\section{Resultados}

Foram calculados cinco complementos alimentares: padrão, proteico, misto 1, misto 2; e sem laticínios, conforme demonstrados na Tabela 1. A composição nutricional (Tabela 2) diferenciou-se de acordo com os ingredientes, sendo que a oferta calórica em uma porção variou entre 203 a 208kcal, enquanto a oferta proteica variou entre 11 a $20 \mathrm{~g}$. Os custos por porção variaram entre $\mathrm{R} \$ 0,91$ a $\mathrm{R} \$ 2,00$.

Tabela 1 - Ingredientes e custos dos complementos alimentares.

\begin{tabular}{|c|c|c|c|}
\hline Complemento alimentar & Ingredientes & Tamanho porção & Custo porção \\
\hline 1 - Padrão & $\begin{array}{l}\text { 30g leite em pó desnatado } \\
20 \mathrm{~g} \text { farinha de aveia } \\
2 \mathrm{ml} \text { óleo de canola }\end{array}$ & $52 \mathrm{~g}$ & $\mathrm{R} \$ 0,91$ \\
\hline 2 - Proteico & $\begin{array}{l}20 \mathrm{~g} \text { whey protein } \\
30 \mathrm{~g} \text { farinha de aveia } \\
2 \mathrm{ml} \text { óleo de canola }\end{array}$ & $52 \mathrm{~g}$ & $\mathrm{R} \$ 2,00$ \\
\hline 3 - Misto 1 & $\begin{array}{l}30 \mathrm{~g} \text { leite em pó desnatado } \\
20 \mathrm{~g} \text { maltodextrina } \\
2 \mathrm{ml} \text { óleo de canola }\end{array}$ & $52 \mathrm{~g}$ & $\mathrm{R} \$ 0,99$ \\
\hline 4 - Misto 2 & $\begin{array}{l}20 \mathrm{~g} \text { leite em pó desnatado } \\
10 \mathrm{~g} \text { whey protein } \\
20 \mathrm{~g} \text { maltodextrina } \\
2 \mathrm{ml} \text { óleo de canola }\end{array}$ & $52 \mathrm{~g}$ & $\mathrm{R} \$ 1,63$ \\
\hline 5-Sem laticínios & $\begin{array}{l}20 \mathrm{~g} \text { albulmina } \\
30 \mathrm{~g} \text { maltodextrina } \\
2 \mathrm{ml} \text { óleo de canola } \\
\end{array}$ & $52 \mathrm{~g}$ & $\mathrm{R} \$ 1,60$ \\
\hline
\end{tabular}

Fonte: Autores (2021).

Tabela 2 - Composição nutricional dos complementos alimentares.

\begin{tabular}{|c|c|c|c|c|c|}
\hline Complemento & Padrão & Proteico & Misto 1 & Misto 2 & Sem laticínios \\
\hline Calorias (kcal) & 203 & 208 & 203 & 205 & 205 \\
\hline Proteínas (g) & 14 & 20 & 11 & 15 & 17 \\
\hline Carboidratos (g) & 29 & 21 & 35 & 30 & 30 \\
\hline Gorduras totais $(\mathrm{g})$ & 3,5 & 5,1 & 2,2 & 2,8 & 2,0 \\
\hline Gordura saturada (g) & 0,5 & 1,1 & 0,3 & 0,6 & 0 \\
\hline Gordura poli-insaturada (mg) & 1040 & 1260 & 580 & 580 & 570 \\
\hline Ômega 3 (mg) & 160 & 170 & 140 & 140 & 140 \\
\hline Fibra $(\mathrm{g})$ & 2,0 & 3,6 & 0 & 0 & 0 \\
\hline Cálcio (mg) & 387,5 & 16 & 377 & 251 & 10 \\
\hline Sódio (mg) & 161 & 48 & 161 & 131 & 33 \\
\hline
\end{tabular}

Fonte: Autores (2021).

A partir dos complementos foram desenvolvidas preparações fortificadas, descritas na Tabela 3. As preparações foram apresentadas em uma cartilha para uso no aconselhamento nutricional (Figura 1). Elaborada em forma de livreto, a cartilha pode ser impressa nas 4 cores, frente e verso ou preto e branco, visando uma linguagem agradável e descomplicada para o fácil entendimento do indivíduo e de seus familiares/cuidadores. 
Tabela 3 - Receitas fortificadas elaboradas com a aplicação dos complementos alimentares.

\begin{tabular}{|c|c|c|c|c|}
\hline Receita & Ingredientes & Medida caseira & $\begin{array}{l}\text { Complementos que } \\
\text { podem ser utilizados }\end{array}$ & $\begin{array}{l}\text { Rendimento } \\
\text { (porção) }\end{array}$ \\
\hline Vitamina ou & $200 \mathrm{ml}$ leite integral & 1 copo grande cheio & 1 (Padrão) & 1 \\
\hline \multirow{4}{*}{ Milkshake de frutas } & $90 \mathrm{~g}$ banana $^{1}$ & 1 unidade média & 2 (Proteico) & \\
\hline & & & 3 (Misto 1) & \\
\hline & & & 4 (Misto 2) & \\
\hline & & & 5 (Sem laticínios) & \\
\hline \multirow[t]{5}{*}{ Creme de abacate } & $400 \mathrm{~g}$ abacate & 1 unidade grande & 1 (Padrão) & 3 \\
\hline & $100 \mathrm{ml}$ leite integral & $1 / 2$ copo & 2 (Proteico) & \\
\hline & 50 g açúcar² & 5 colheres de sopa & 3 (Misto 1) & \\
\hline & $20 \mathrm{~g}$ cacau & 4 colheres de sopa cheias & 4 (Misto 2) & \\
\hline & & & 5 (Sem laticínios) & \\
\hline \multirow{3}{*}{$\begin{array}{l}\text { Mingau de amido de } \\
\text { milho }\end{array}$} & $250 \mathrm{ml}$ leite integral & 1 caneca & 3 (Misto 1) & 1 \\
\hline & $15 \mathrm{~g}$ amido de milho & 1 colher de sopa cheia & 4 (Misto 2) & \\
\hline & $10 \mathrm{~g}$ açúcar $^{2}$ & 1 colher de sopa cheia & & \\
\hline \multirow[t]{3}{*}{ Mingau com aveia } & $250 \mathrm{ml}$ leite integral & 1 caneca & 1 (Padrão) & 1 \\
\hline & $52 \mathrm{~g}$ complementos 1 ou 2 & 1 colher de sopa cheia & 2 (Proteico) & \\
\hline & $10 \mathrm{~g}$ açúcar $^{2}$ & 1 colher de sopa cheia & & \\
\hline \multirow[t]{3}{*}{ Picolé de iogurte } & $150 \mathrm{~g}$ iogurte natural integral & 1 unidade & 3 (Misto 1) & 2 \\
\hline & $10 \mathrm{~g}$ açúcar ${ }^{2}$ & 1 colher de sopa & 4 (Misto 2) & \\
\hline & $5 \mathrm{~g} \mathrm{cacau}^{3}$ & 1 colher de sopa & 5 (Sem laticínios) & \\
\hline \multirow{3}{*}{$\begin{array}{l}\text { Picolé de leite de } \\
\text { coco }\end{array}$} & 140 banana $^{4}$ & 2 unidades pequenas & 3 (Misto 1) & 2 \\
\hline & $100 \mathrm{ml}$ leite de coco & $1 / 2$ copo & 4 (Misto 2) & \\
\hline & $15 \mathrm{~g}$ açúcar $^{2}$ & $1 \frac{1}{2}$ colheres de sopa & 5 (Sem laticínios) & \\
\hline
\end{tabular}

${ }^{1}$ Pode ser substituída por frutas de preferência. ${ }^{2}$ Pode ser substituído por adoçante (stévia) à gosto. ${ }^{3}$ Pode ser substituído por 1 colherzinha de café de suco artificial de fruta. ${ }^{4}$ Pode ser substituída por manga ou morango.

Fonte: Autores (2021).

Figura 1 - Cartilha para orientação nutricional das receitas elaboradas com a aplicação dos complementos alimentares.

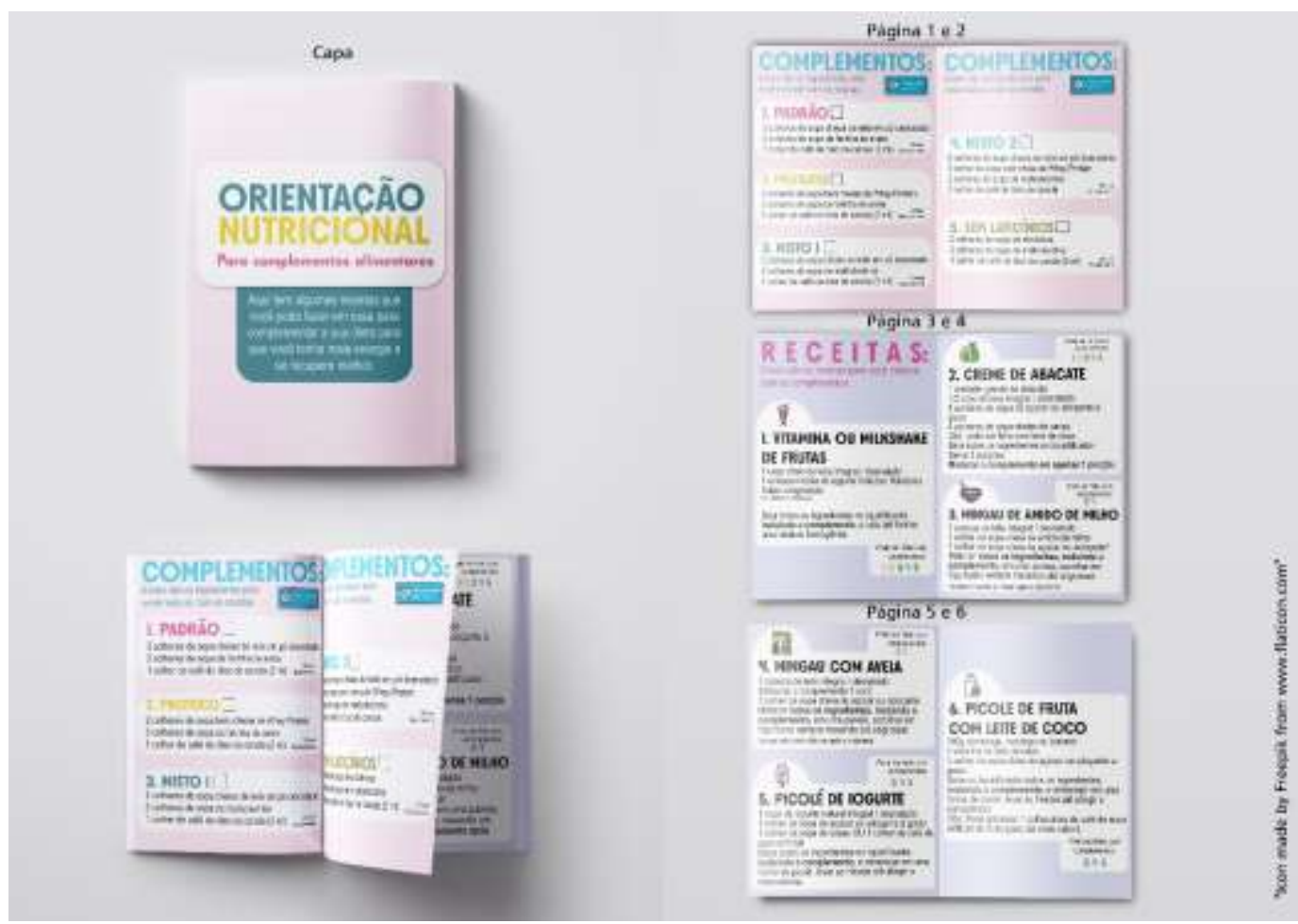

Fonte: Autores (2021). 


\section{Discussão}

As recomendações dietéticas para idosos variam de acordo com o estado nutricional. Para idosos com baixo peso, o valor calórico deve atingir de 32 a 38kcal $/ \mathrm{kg}$ de peso atual/dia, e $1 \mathrm{~g}$ de proteínas $/ \mathrm{kg}$ de peso atual $/$ dia, podendo alcançar até 2,0g/kg de peso atual/dia em idosos com desnutrição ou comorbidades. Recomenda-se a oferta de suplementação de pelo menos 400kcal e 30g de proteína por dia, por no mínimo 1 mês após a alta hospitalar (Volkert et al., 2019). Sugere-se, ainda, ofertar 25 a 30g de proteína por refeição para melhor a resposta anabólica (Horie et al., 2019). A OMS aconselha a suplementação de 400 a $600 \mathrm{Kcal} /$ dia para idosos em risco nutricional ou em desnutrição, acompanhada de monitoramento nutricional (WHO, 2019).

A suplementação auxilia na redução de complicações, mortalidade e tempo de internação hospitalar, contribuindo para a redução de custos (Horie et al., 2019; Elia et al., 2016). Uma dieta hiperproteica contribui para preservação da massa muscular, promovendo melhor funcionalidade no envelhecimento (Horie et al., 2019; Bauer et al., 2013). A suplementação proteica pode ser associada com treinamento resistido, melhorando a mobilidade em idosos com risco de sarcopenia (Liao et al., 2019).

Os complementos alimentares desenvolvidos neste trabalho atingem características preconizadas pelos guidelines de nutrição para idosos (WHO, 2019; Horie et al.,2019; Volkert et al., 2019), havendo indicação para consumo duas vezes ao dia. Cada um dos complementos apresenta características particulares.

O complemento alimentar padrão atingiu a recomendação de calorias e proteínas suplementares, apresentou um custo menor do que as outras opções e seus ingredientes são facilmente encontrados. Parte das proteínas deste complemento são advindas do leite, com alta biodisponibilidade, e da aveia, com limitação de aminoácidos essenciais e menor biodisponibilidade (Tirapegui, Castro \& Rossi, 2005). No entanto, a aveia é fonte de fibras de alta viscosidade, que aumentam a sensação de saciedade (Martinez-Villaluenga \& Penas, 2017). Essas fibras têm efeito modulador do trânsito e da microbiota intestinal, e apresentam benefícios metabólicos (Deehan et al., 2018).

O complemento alimentar proteico destaca-se pelo perfil de proteínas do soro do leite (whey protein). Ele é comumente utilizado por promover a síntese proteica muscular, sendo fonte de aminoácidos essenciais e de cadeia ramificada de alta biodisponibilidade (Tirapegui, Castro \& Rossi, 2005; Jäger, 2017). Apesar da ótima composição proteica, este complemento apresentou maior custo.

O complemento misto 1 pode ser uma melhor opção de carboidratos. A maltodextrina é um polímero de glicose sem fibras solúveis, o que poderia reduzir a sensação de plenitude gástrica em relação ao complemento padrão (Hofman, Van Buul $\&$ Brouns, 2016; Cardoso, Seabra \& de Souza, 2017). Mesmo que ofertada duas vezes ao dia, não alcançaria as recomendações de suplementação de proteínas, no entanto, atingiu as recomendações calóricas e apresentou boa aplicabilidade em todas as receitas.

O complemento alimentar misto 2 apresenta vantagem quanto à composição proteica, tendo parte das proteínas advindas do whey protein, o que amplia a oferta de aminoácidos essenciais leucina, isoleucina e valina (Tirapegui, Castro \& Rossi, 2005; Jäger et al., 2017). Seu custo ficou em valor intermediário e teve boa aplicabilidade em todas as receitas.

O complemento alimentar sem laticínios considera a possibilidade de deficiência de lactase na população idosa (Schiller, 2020). Seu custo foi intermediário, tendo em sua composição a albumina, proteína de alta biodisponibilidade e custo mediano (Tirapegui, Castro \& Rossi, 2005). A albumina apresentou aplicabilidade apenas em algumas receitas, não podendo ser aquecida em função da desnaturação proteica.

Uma alimentação adequada contribui para a saúde óssea, sendo a ingestão de cálcio um fator chave para a prevenção e tratamento da osteoporose em idosos (Coronado-Zarco et al., 2019; Radominski, 2017), sendo que a recomendação de cálcio para idosos é de 1200mg/dia (Coronado-Zarco et al., 2019; França \& Martini, 2014). Os complementos elaborados com leite 
em pó apresentaram elevadas quantidades de cálcio. A oferta do complemento alimentar padrão, duas vezes ao dia, seria capaz de suprir pelo menos $60 \%$ das necessidades de cálcio. Outros benefícios do consumo de laticínios no envelhecimento são a redução da fragilidade e prevenção da sarcopenia (Cuesta-Triana et al., 2019).

Buscou-se a possibilidade de adaptação a indivíduos com diabetes e disfagia, condições comuns em pacientes idosos. Nesse sentido, os complementos alimentares padrão e proteico contam com efeitos reguladores das fibras da aveia sobre a glicemia (Deehan et al., 2018; Sociedade Brasileira de Diabetes, 2019), podendo ser utilizados por pacientes diabéticos. Já os complementos com maltodextrina, podem ser recomendados pelo nutricionista conforme individualidade do paciente, se incluídos em um contexto alimentar saudável (Hofman, Van Buul \& Brouns, 2016; Sociedade Brasileira de Diabetes, 2019). Todas as receitas se adaptam ao uso de adoçantes naturais/artificiais no preparo. É importante ressaltar que vitaminas, mingaus e cremes exigem pouco esforço para mastigação, e podem ser ajustadas de acordo com o grau da disfagia com o uso de espessantes.

Os complementos alimentares apresentam desvantagem por não serem acrescidos de vitaminas e minerais. Entretanto não levam insumos industriais normalmente utilizados para cor, sabor, estabilidade e conservação ao produto final. Ainda, a praticidade e pouca manipulação também são vantagens dos suplementos industrializados. Apesar disso, os complementos produzidos neste estudo alcançaram adequada composição de energia e macronutrientes, bem como o destaque para o micronutriente cálcio. As receitas fortificadas ainda permitirem o uso de ingredientes sazonais e adaptação às preferências e cultura do indivíduo.

Embora a comparação de valores e composição nutricional possa ser observada em outros estudos realizados no Brasil (Dias \& Fortes, 2019), compreendemos que as preparações elaboradas neste estudo apresentam um processo de produção diferente do industrial, com vantagens e desvantagens que devem ser avaliadas individualmente pelo profissional de saúde para a indicação da opção mais adequada.

O uso de cartilhas pode ser uma prática positiva no incentivo ao autocuidado e a aderência a terapia nutricional (de Sousa Gonçalves et al., 2019). Estudo realizado com pacientes em perioperatório de cirurgia bariátrica (Barros et al., 2020) observou maior efetividade na orientação e manutenção na atitude positiva com a utilização da cartilha quando comparada ao método de rotina. Em pacientes da atenção básica com diabetes mellitus (de Carvalho Torres \& de Paula, 2019) a orientação com a cartilha alcançou os objetivos de educação para o autocuidado. Alguns estudos (de Carvalho Torres \& de Paula, 2019; Santos et al., 2020) que demonstram o uso de cartilhas avaliam a eficácia do material elaborado a partir de sua aplicabilidade. O presente estudo prevê essa avaliação de aplicabilidade em trabalhos futuros.

Foram encontrados poucos artigos científicos com objetivos semelhantes ao nosso estudo. Um grupo de pesquisa brasileiro realizou análise descritiva de suplementos alimentares para pacientes oncológicos (Garófolo \& Rezende, 2010). Os mesmos autores realizaram um ensaio clínico prospectivo não controlado, ofertando suplementação a crianças e adolescentes com câncer, em que destacaram a viabilidade do uso dos suplementos artesanais na recuperação do estado nutricional (Alves et al., 2010). Outro estudo recente, realizado no Distrito Federal, avaliou sensorialmente suplementos artesanais utilizando ingredientes como clara de ovo, beterraba, banana, aveia, cacau, entre outros. Os autores realizaram testes com adultos jovens e saudáveis, observaram bons resultados de aceitação das fórmulas elaboradas e redução significativa dos custos (Dias \& Fortes, 2019).

\section{Considerações Finais}

Neste trabalho foram elaboradas opções de complementos alimentares, demonstrando ser possível alcançar as recomendações para suplementação de idosos em risco nutricional, com opções caseiras e de mais fácil acesso. Viabilizando a aplicação prática das receitas fortificadas, desenvolveu-se uma cartilha educativa para o aconselhamento nutricional. 
Considerando o cenário econômico e de saúde do Brasil, trata-se de um trabalho relevante visto que possibilita o uso de complementos com menor custo, garantindo a sequência à terapia nutricional domiciliar e contribuindo no combate à desnutrição.

Em consideração à relevância do tema abordado, sugerimos mais estudos em que sejam realizadas pesquisas de análise sensorial das preparações elaboradas, bem como avaliar as habilidades culinárias para elaboração das preparações propostas pela população idosa. O instrumento de orientação nutricional elaborado deverá ser aplicado em unidades hospitalares com o público alvo.

\section{Agradecimentos}

Agradecemos a Thais Cauduro, chefe do Serviço de Nutrição do Hospital Universitário de Santa Maria, pelo apoio ao projeto e à sua execução.

\section{Referências}

Alves, F. R., Garófolo, A., Maia, P. D. S., Nóbrega, F. J. D., \& Petrilli, A. S. (2010). Suplemento artesanal oral: uma proposta para recuperação nutricional de crianças e adolescentes com câncer. Revista de Nutrição, 23(5), 731-744.

Barros, L. M., Gomes, F. A. D. V., Carneiro, F. N., Galindo Neto, N. M., Frota, N. M., \& Caetano, J. Á. (2020). Knowledge and attitude of candidates to gastroplasty about perioperative: randomized clinical trial. Revista Brasileira de Enfermagem, 73(6), e20180869.

Bauer, J., Biolo, G., Cederholm, T., Cesari, M., Cruz-Jentoft, A. J., Morley, J. E., Phillips, S., Sieber, C., Stehle, P., Teta, P., Visvanathan, R., Volpi, E., \& Boirie, Y. (2013). Evidence-based recommendations for optimal dietary protein intake in older people: a position paper from the PROT-AGE Study Group. Journal of the american Medical Directors association, 14(8), 542-559.

Cardoso, M., Seabra, T. T. P., \& de Souza, E. B. (2017). Dextrose, Maltodextrina e Waxy Maize: principais diferenças na composição, mecanismo de ação e recomendações para o desempenho esportivo. Cadernos UniFOA, 12(33), 101-109.

Cederholm, T., Barazzoni, R., Austin, P., Ballmer, P., Biolo, G., Bischoff, S. C., Compher, C., Correia, I., Higashiguchi, T., Holst, M., Jensen, G. L., Malone, A., Muscaritoli, M., Nyulasi, I., Pirlich, M., Rothenberg, E., Schindler, K., Schneider, S. M., de van der Schueren, M. A. E., Sieber, C., Valentini, L., Yu, J. C., Van Gossum, A., \& Singer, P. (2017). ESPEN guidelines on definitions and terminology of clinical nutrition. Clinical nutrition, 36(1), 49-64.

Coronado-Zarco, R., de León, A. O. G., García-Lara, A., Quinzaños-Fresnedo, J., Nava-Bringas, T. I., \& Macías-Hernández, S. I. (2019). Nonpharmacological interventions for osteoporosis treatment: Systematic review of clinical practice guidelines. Osteoporosis and sarcopenia, 5(3), 69-77.

Cuesta-Triana, F., Verdejo-Bravo, C., Fernández-Pérez, C., \& Martín-Sánchez, F. J. (2019). Effect of milk and other dairy products on the risk of frailty, sarcopenia, and cognitive performance decline in the elderly: a systematic review. Advances in Nutrition, 10(suppl_2), S105-S119.

De Carvalho Torres, H., \& de Paula, D. V. (2019). Avaliação da cartilha para orientação da prática do autocuidado em Diabetes Mellitus [Evaluation of a primer giving guidance on Diabetes Mellitus self-care][Evaluación del manual para orientación de la práctica del autocuidado en Diabetes Mellitus]. Revista Enfermagem UERJ, 27, 7722.

De Sousa Gonçalves, M., Celedônio, R. F., Targino, M. B., de Oliveira Albuquerque, T., Flauzino, P. A., Bezerra, A. N., Albuquerque, N. V., \& Lopes, S. C. (2019). Construção e validação de cartilha educativa para promoção da alimentação saudável entre pacientes diabéticos. Revista Brasileira em Promoção da Saúde, 32 .

Deehan, E. C., Duar, R. M., Armet, A. M., Perez-Munoz, M. E., Jin, M., \& Walter, J. (2018). Modulation of the gastrointestinal microbiome with nondigestible fermentable carbohydrates to improve human health. Bugs as Drugs: Therapeutic Microbes for the Prevention and Treatment of Disease, 453483.

Dias, M. C., \& Fortes, R. C. (2019). Análise sensorial de suplementos nutricionais artesanais utilizados como alternativa terapêutica para idosos desnutridos. Nutrição Brasil, 18(1), 21-28.

Elia, M., Normand, C., Laviano, A., \& Norman, K. (2016). A systematic review of the cost and cost effectiveness of using standard oral nutritional supplements in community and care home settings. Clinical nutrition, 35(1), 125-137.

França, N. A. G., \& Martini, L. A. (2014). Cálcio - Funções Plenamente Reconhecidas de Nutrientes. ILSI Brasil. 2018. https://ilsibrasil.org/publication/funcoes-plenamente-reconhecidas/

Garófolo, A., Alves, F. R., \& Rezende, M. A. D. C. (2010). Suplementos orais artesanais desenvolvidos para pacientes com câncer: análise descritiva. Revista de Nutrição, 23(4), 523-533.

Hofman, D. L., Van Buul, V. J., \& Brouns, F. J. (2016). Nutrition, health, and regulatory aspects of digestible maltodextrins. Critical reviews in food science and nutrition, 56(12), 2091-2100. 
Horie, L. M., Barrére, A. P. N., Castro, M. G., Liviera, A. M. B., Carvalho, A. M. B., \& Pereira, A. (2019). Diretriz BRASPEN de terapia nutricional no paciente com câncer. BRASPEN J, 34(Supl 1), 2-32.

Jäger, R., Kerksick, C. M., Campbell, B. I., Cribb, P. J., Wells, S. D., Skwiat, T. M., Purpura, M., Ziegenfuss, T. N., Ferrando, A. A., Arent, S. M., SmithRyan, A. E., Stout, J. R., Arciero, P. J., Ormsbee, M. J., Taylor, L. W., Wilborn, C. D., Kalman, D. S., Kreider, R. B., Willoughby, D. S., Hoffman, J. R., Krzykowsk, J. S., \& Antonio, J. (2017). International society of sports nutrition position stand: protein and exercise. Journal of the International Society of Sports Nutrition, 14(1), 1-25.

Liao, C. D., Chen, H. C., Huang, S. W., \& Liou, T. H. (2019). The role of muscle mass gain following protein supplementation plus exercise therapy in older adults with sarcopenia and frailty risks: a systematic review and meta-regression analysis of randomized trials. Nutrients, 11(8), 1713.

Martinez-Villaluenga, C., \& Penas, E. (2017). Health benefits of oat: Current evidence and molecular mechanisms. Current Opinion in Food Science, 14, 2631.

Martins, A. L. C., Bertin, R. L., Calao, K. M. F. N., \& Medeiros, C. O. (2020). Habilidades culinárias de idosos praticantes de atividades aquáticas. Research, Society and Development, 9(2), e31921981.

Radominski, S. C., Bernardo, W., Paula, A. P. D., Albergaria, B. H., Moreira, C., Fernandes, C. E., Castro, C. H. M., C. A. F., Domiciano, D. S., Mendonça, L. M. C., Pompei, L. M., Bezerra, M. C., Loures, M. A. R., Wender, M. C. O., Lazaretti-Castro, M., Pereira, R. M. R., Maeda, S. S., Szejnfeld, V. L., \& Borba, V. Z. (2017). Brazilian guidelines for the diagnosis and treatment of postmenopausal osteoporosis. Revista brasileira de reumatologia, 57, s452-s466.

Santos, S. L. F., Mormino, K. B. N. T., da Silva Alves, H. H., Otoni, K. M., Pessoa, C. V., de Sousa Magalhães, A. R., Silveira, J. E. S., de Lima, J. P., Souza, M. M. S., \& Néri, E. D. R. (2020). Uso seguro de medicamentos em gestantes: construção e validação de uma cartilha educativa. Revista Eletrônica Acervo Saúde, (49), e3274-e3274.

Schiller, L. R. (2020). Maldigestion Versus Malabsorption in the Elderly. Current Gastroenterology Reports, 22, 1-8.

Silva, V. S. D., Souza, I., Silva, D. A. S., Barbosa, A. R., \& Fonseca, M. D. J. M. D. (2018). Evolução e associação do IMC entre variáveis sociodemográficas e de condições de vida em idosos do Brasil: 2002/03-2008/09. Ciência \& Saúde Coletiva, 23, 891-901.

Sociedade Brasileira de Diabetes (SBD) (2019). Diretrizes da Sociedade Brasileira de Diabetes 2019-2020. Clannad Editora Científica.

Tirapegui, J., Castro, I. A., \& Rossi, L. (2005). Biodisponibilidade de proteínas. Cozzolino, SMF. Biodisponibilidade de Nutrientes. Manole.

Volkert, D., Beck, A. M., Cederholm, T., Cruz-Jentoft, A., Goisser, S., Hooper, L., Kiesswetter, E., Maggio, M., Raynaud-Simon, A., Sieber, C. C., Sobotka, L., van Asselt, D., Wirth, R., \& Bischoff, S. C. (2019). ESPEN guideline on clinical nutrition and hydration in geriatrics. Clinical nutrition, 38(1), 10-47.

World Health Organization (WHO). (2017). Integrated care for older people: guidelines on community-level interventions to manage declines in intrinsic capacity. https://apps.who.int/iris/handle/10665/258981.

World Health Organization (WHO). (2019). Integrated care for older people (ICOPE): guidance for person-centred assessment and pathways in primary care (No. WHO/FWC/ALC/19.1). World Health Organization. 